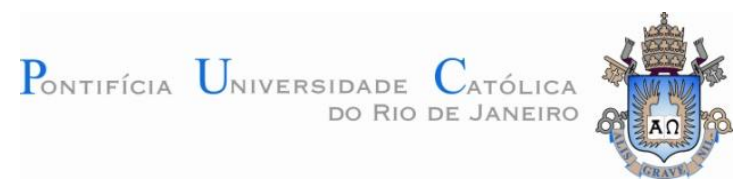

Victor Barbosa Martin

\title{
Melhoria da confiabilidade metrológica na inspeção de componentes usinados com aplicação na indústria \\ nuclear
}

Dissertação de Mestrado

Dissertação apresentada como requisito parcial para obtenção do título de Mestre pelo Programa de Pós-Graduação em Metrologia da PUC-Rio (Área de Concentração: Metrologia para Qualidade e Inovação).

Orientador:

Professor Dr. Mauricio Nogueira Frota Programa de Pós-Graduação em Metrologia da Pontifícia Universidade Católica do Rio de Janeiro (PosMQI/PUC-Rio)

Co-Orientador:

Professor Dr. Gustavo Daniel Donatelli

Programa de Pós-Graduação em Metrologia da Universidade Federal de

Santa Catarina (PosMCI/UFSC)

Rio de Janeiro, 14 abril de 2010 


\section{Melhoria da confiabilidade metrológica na inspeção de componentes usinados com aplicação na indústria}

nuclear

Dissertação apresentada como requisito parcial para obtenção do título de Mestre em Metrologia pela PUCRio. Aprovada pela Comissão Examinadora abaixo assinada.

Professor Dr. Mauricio Nogueira Frota

Orientador

Programa de Pós-Graduação em Metrologia da (PósMQI/PUC-Rio)

Pontifícia Universidade Católica do Rio de Janeiro

Professor Dr. Gustavo Daniel Donatelli Co-Orientador Programa de Pós-Graduação em Metrologia da (Pós MCI/UFSC) Universidade Federal de Santa Catarina

Prof. Dr. Carlos Roberto Hall Barbosa Programa de Pós-Graduação em Metrologia da (PósMQI/PUC-Rio) Pontifícia Universidade Católica do Rio de Janeiro

Dr. Maurício de Campos Porath Fundação Centro de Referência em Tecnologias Inovadoras (CERTI)

Prof. José Eugênio Leal Coordenador Setorial da Pós-Graduação Centro Técnico Científico (PUC-Rio) 
Todos os direitos reservados. É proibida a reprodução total ou parcial do trabalho sem autorização da universidade, do autor e do orientador.

\section{Victor Barbosa Martin}

Graduou-se em Engenharia Mecânica (1989), (habilitação industrial) pelo CEFET-CSF (Centro Federal de Educação Tecnológica Celso Suckow da Fonseca) no Rio de Janeiro. Atualmente é Engenheiro da INB (Indústrias Nucleares do Brasil).

Ficha Catalográfica

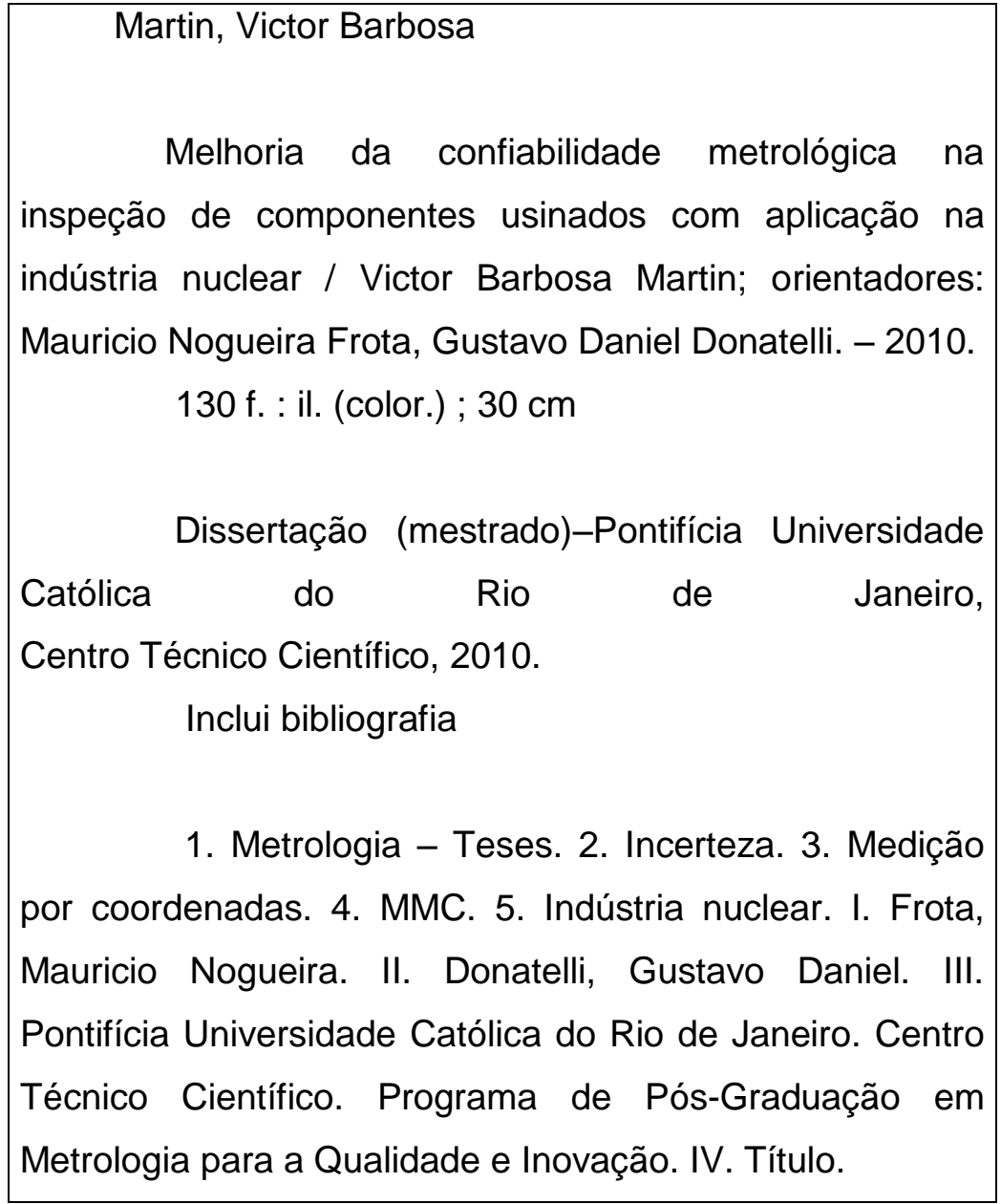

CDD: 389.1 
Aos nossos antepassados, dando continuidade à manifestação da curiosidade e à busca do conhecimento humano. 


\section{Agradecimentos}

À INB, empresa para a qual trabalho, que pelo seu programa de incentivo a educação proporcionou esta oportunidade.

Ao Professor Gustavo Donatelli pelo inteligente apoio técnico e por ter facilitado o acesso ao Laboratório de Máquinas de Medir Coordenadas da Fundação CERTI, fundamental no desenvolvimento da pesquisa.

E principalmente ao Prof. Maurício N. Frota, que acreditou em minha idéia, e apesar de todos os percalços vivenciados alimentou-me de estímulo, permitindome encontrar forças para prosseguir na busca do meu objetivo. 


\section{Resumo}

Martin, Victor Barbosa; Frota, Mauricio Nogueira. Melhoria da confiabilidade metrológica na inspeção de componentes usinados com aplicação na indústria nuclear. Rio de Janeiro, 2010. 130 p. Dissertação de Mestrado - Programa de Pós-Graduação em Metrologia (Área de Concentração: Metrologia para Qualidade e Inovação). Pontifícia Universidade Católica do Rio de Janeiro.

A presente pesquisa de mestrado contribui para a melhoria da confiabilidade metrológica de componentes usinados, utilizados na indústria nuclear para produção de energia elétrica, produzidos com reduzidas tolerâncias definidas em projeto. O trabalho é de interesse da empresa Indústrias Nucleares do Brasil (INB), que atua na cadeia produtiva do urânio e produz o elemento combustível para as usinas nucleares de Angra, pois aprimora a analise do processo de medir. Cumprindo o objetivo da dissertação e fazendo uso da técnica de medição por coordenadas, foi desenvolvida uma metodologia para avaliação do processo de inspeção dimensional de componentes usinados da indústria nuclear. A motivação é a melhoria da confiabilidade metrológica requerida na inspeção, agregando conhecimento e inovação tecnológica. O trabalho incorpora uma análise das incertezas associadas ao processo de medição por coordenadas de componentes usinados. A metodologia utiliza a pesquisa bibliográfica e a experimental e, à luz dos métodos de avaliação de incertezas baseados numa formulação estatística, avalia processos de medição de interesse da indústria nuclear. Dentre os resultados são caracterizadas as influências na medição da característica objeto da medição; é avaliada a divergência da medição em relação a um padrão e desenvolvida uma ferramenta para a avaliação e melhoria do processo de medição. Como conclusão, o trabalho contribui com o aprimoramento da técnica de medição por coordenadas incorporando inovações tecnológicas na melhoria da qualidade dos processos de inspeção dimensional.

\section{Palavras-chave}

Metrologia; incerteza; medição por coordenadas; MMC; indústria nuclear. 


\section{Abstract}

Martin, Victor Barbosa; Frota, Mauricio Nogueira (Advisor). Improvement of the metrological reliability in the inspection of machined components with applications in the nuclear industry. Rio de Janeiro, 2010. 130 p. MSc. Dissertation - Programa de Pós-Graduação em Metrologia (Área de Concentração: Metrologia para Qualidade e Inovação). Pontifícia Universidade Católica do Rio de Janeiro.

This Master's Thesis contributes to the improvement of metrological reliability of machined components that are used in the nuclear industry for the production of electrical energy and which are produced with reduced tolerances defined in the project. The work is of interest to the company Indústrias Nucleares do Brasil (INB), which operates in production of the uranium fuel assemblies for nuclear power plants in Angra, improving the analysis of the measuring process. The dissertation fulfill the goal, by the development of a methodology to use in evaluation of coordinate measuring technique that is used in the process of dimensional inspection of machined components for the nuclear industry. The motivation is to improve the metrological reliability required in the inspection, adding knowledge and technological innovation. The work incorporates an analysis of uncertainties associated with the measurement process by coordinates, of machined components. The methodology uses bibliographic research and experimental methods of evaluation of uncertainty based on a statistical formulation, in measurement procedures of interest to the nuclear industry. In the results are characterized the influences of the measurement on the object characteristic of measurement, the divergence is evaluated by measuring against a standard. It was developed a tool to evaluate and improve the measurement process. In conclusion, the study contributes to the improvement of the coordinate measuring technique incorporating technological innovations, improving the quality of procedures for dimensional inspection.

\section{Keywords}

Metrology; uncertainty; coordinate measuring; MMC; nuclear industry. 


\section{Sumário}

1 . Introdução 14

1.1. O ambiente da pesquisa 14

1.1.1. O controle dimensional de componentes usinados 15

1.1.2. Particularidades do controle dimensional da INB 17

1.1.3. Garantia da qualidade das medições 18

1.1.4. Práticas da garantia da qualidade das medições na INB 20

1.2. Foco da pesquisa 21

1.3. Objetivos da pesquisa 22

1.3.1. Objetivo geral 22

1.3.2. Objetivos específicos 23

1.4. Metodologia 24

1.5. Estrutura da dissertação 25

2. Fundamentos da medição por coordenadas 27

2.1. Fontes de erros em medição por coordenadas 28

2.1.1. A máquina de medir por coordenadas 29

2.1.1.1. A estrutura 29

2.1.1.2. O sistema de apalpação 30

2.1.2. Ambiente de medição 32

2.1.3. A peça objeto da medição 32

2.1.4. Estratégia de medição 33

2.1.5. O operador 35

2.2. Avaliação da confiabilidade metrológica de processos por MMC 36

2.2.1. Metrologia para garantia da qualidade 37

2.2.2. O manual de análise dos sistemas de medição 39

2.2.3. A incerteza de medição 41

2.2.4. Incerteza em medição por coordenadas 42

3. Confiabilidade da inspeção de componentes usinados 45

3.1. Características dos componentes usinados sob avaliação 46 
3.1.1. Considerações funcionais das características do estudo

3.2. A construção de um padrão geométrico

3.2.1. Definição das características a calibrar 50

3.2.2. Seleção e calibração do padrão 51

3.2.3. A estratégia de medição 53

3.2.4. Estratégia da calibração do padrão 54

3.2.5. Estratégia da medição industrial 54

3.3. Metodologia para a realização e implantação dos estudos

na MMC INB 57

3.3.1. Estudo da estabilidade e tendência 59

3.3.2. Estudo de repetitividade 60

4. Resultados experimentais 61

4.1. Controle aplicado à estrutura de medição 62

4.2. Estudo de repetitividade do processo de medição 62

4.2.1. Planeza (AY1) da superfície de referência $T$

4.2.2. Diâmetro (BN) e posição (BE) dos furos $\mathrm{S} 1$ e S2 64

4.2.3. Paralelismo (CZ) em relação a referência "T" e espessura (BA) 66

4.2.4. Diâmetro $(B M)$ e posição $(B D)$ dos furos $P$ de fixação

dos tubos guia 68

4.2.5. Resumo dos resultados dos estudos de repetitividade $\quad 70$

4.3. Estudo de estabilidade e tendência do processo de medição $\quad 70$

4.3.1. Estabilidade da temperatura 71

4.3.2. Planeza (AY1) da superfície de referência " $T$ " 72

4.3.3. Diâmetro (BN) e posição (BE) dos furos $\mathrm{S} 1$ e S2 73

4.3.4. Paralelismo(CZ) e espessura(BA) das sapatas

em referência à " $T$ "

4.4. Resumo dos resultados do estudo de estabilidade 81

4.5. Correlação com a temperatura 82

5 . Discussão e oportunidade de melhoria 84

5.1. Análise da medição na INB 86

5.1.1. Análise dos pontos fortes 86

5.1.2. Análise dos pontos fracos 87 
5.1.3. Análise das oportunidades para melhorar a gestão da medição tridimensional na INB 87

5.1.4. Oportunidades para melhorar a medição do Bocal Inferior

6. Conclusões

7 . Bibliografia

8. Siglas e abreviações

9. Anexos 


\section{Lista de figuras}

Figura 1. Instalações da Fábrica de Combustível Nuclear (FCN1) da INB 15

$\begin{array}{ll}\text { Figura 2. Fluxo de produção de componente usinado } & 18\end{array}$

Figura 3. Medição do componente Bocal Inferior (BI) na INB 21

Figura 4. Erros geométricos associados à operação de uma

máquina de medir por coordenadas $\quad 30$

Figura 5. O processo de medição e suas consequências 38

Figura 6. Bocal Inferior do Elemento Combustível (BI do EC) 46

Figura 7. Estrutura do Elemento Combustível 47

Figura 8. Elemento Combustível 16 NGF e uma vareta do EC em corte 47

Figura 9. MMC utilizada para calibrar a peça padrão na CERTI 49

Figura 10. Localização, no BI, das características do estudo 51

Figura 11. Peça BI fixada em dispositivo 55

Figura 12. Medição no modo scanning do furo S, na INB 55

Figura 13. Seqüência de medição do furo P, por apalpação, na INB 56

Figura 14. Seqüência de medição da característica CZ, na INB 56

Figura 15. Seqüência de medição da característica AY1, na INB 57

Figura 16. Esquema da estrutura física do laboratório de medição 62

Figura 17. Região de planeza AY1 63

Figura 18. Característica de planeza AY1 64

Figura 19. Localização dos furos de posicionamento S1 e S2 64

Figura 20. Característica de dimensão BN, furos S1 e S2 65

Figura 21. Característica de posição BE $\quad 65$

Figura 22. Localização da base das sapatas de apoio do BI 66

$\begin{array}{ll}\text { Figura 23. Característica de paralelismo CZ } & 67\end{array}$

Figura 24. Característica de dimensão BA $\quad 67$

Figura 25. Localização dos furos de fixação dos tubos guia 68

$\begin{array}{ll}\text { Figura 26. Característica de diâmetro BM } & 68\end{array}$

Figura 27. Característica de posição BD1 69

Figura 28. Característica de posição BD2 69

Figura 29. Tempo entre repetições 
Figura 30. Variação da temperatura $\quad 72$

Figura 31. Característica de planeza AY1 73

Figura 32. Diâmetro BN do furo S1 74

Figura 33. Diâmetro BN do furo S2 74

Figura 34. Característica de posição BE do furo S1 75

Figura 35.Característica de posição BE do furo S1 76

Figura 36. Característica de posição BE do furo S2 76

Figura 37. Controle do diâmetro BM do furo P4 77

Figura 38.Controle do diâmetro BM do furo P2 78

Figura 39. Desvio vetorial da característica de posição BD1 do furo P4 79

Figura 40. Desvio vetorial da característica de posição BD1 do furo P2 79

Figura 41. Desvio vetorial da característica de posição BD2 do furo P4 80

Figura 42. Desvio vetorial da característica de posição BD2 do furo P2 81

Figura 43. Característica geométrica de planeza AY1 82

Figura 44. Característica geométrica de posição BE no furo S1 83

Figura 45. Influência da variação de temperatura no resultado da medição 83 


\section{Lista de tabelas}

Tabela 1. Tabela de similaridade, fonte ISO TS 15530-3 51

$\begin{array}{ll}\text { Tabela 2. Resultados de repetitividade } & 70\end{array}$

Tabela 3. Resultados de estabilidade $\quad 82$ 